\title{
Verhandlungen der Sociedad espaňola de Dermatologia y Sifiliografia.
}

Sitzungen Februar-März 1913.

\author{
Azua, Juan de. Zur Behandlang der Urethritis gonor- \\ rhoica acuta anterior. \\ 1 mal täglich Spülung der vorderen Harnröhre mittels doppel-
} läufiger Kanüle oder biegsamem Katheter bei mäßigem Druck mit 300 bis $500 \mathrm{ccm}$ einer möglichst warmen Lösung von Kal. permang. 1:10.000 bis $0.5: 1000$. 3 mal täglich Einspritzung von Argent. nitr. $1: 20.000$ bis $01:$ : 400. Dauer der Behandlung 4-6 Wochen. Unter etwa 50 so be. handelten Patienten 9 Mißerfolge. litis chen.

Azua, Juan de. Reinfektion bei einem tertiär Syphi-

36 jähriger Mann, 1897 Lues, die nur ungenügend behandelt wurde, Jnli 1913 Lues III, papulo-gummöses Syphilid am Kopf, Behandlung mit 6 Injektionen von 01 . cinereum 0.08 und 2 intravenösen Injektionen von 0.3 und 0.4 Salvarsan. Was erman nositiv. Nach 9 Monaten Primäraffekt mit folgender Angina specifica und papulösem Exanthem. W a s s ermann positiv. Verschwinden des Exanthems unter Behandlung mit 0l. cinereum und Neosalvarsan 1 mal $0 \cdot 45$.

A. nimmt an, daßj die Spirochaeten der 1. Infektion nicht genügend Antikörper zu bilden vermocht hatten, um die 2. Infektion mit einem neven virulenten Spirochaetenstamm zu verhindern.

Aja, Sainz de. Nachschübe vou Lichen scrophulosorum im Verlauf eines mit Tuberkulin behandelten Lupus.

7 Jahre alt, Lupus der Nase, Wange und Lippen mit Beteiligung der Schleimhäute seit Januar 1911. Behandlung mit Pyrogallol, Perubalsam, Silbernitrat ohne Erfolg. Oktober 1912 Beginn mit Tuberkulinbehandlung, es bestand damals nur ein geringer Lichen scrophulosorum am Rücken. Im Verlauf der $1 \frac{1}{2}$ Monate dauernd Tuberkulinbehandlung, 0.005-0.1 $\mathrm{mg}$, Aufschießen massenhafter frischer Lichenelemente auf Brust, Lende und Bauch ohne Fieber oder sonstige Allgemeinerscheinuugen. Lupus nicht beeinflußt.

A. ist der Ansicht, daß durch das Tuberkulin latent bereits vorhandene Herde klinisch manifest gemacht wurden.

Covisa, José S. Syphilis der Lunge.

37 Jahre alter Mann, seit 18 Jahren Lues, ungenügend behandelt. Seit einem Jahr Husten, blutiger Auswurf und sehr heftige Dyspnoe Zuerst für Tuberkulose gehalten und ohne Erfolg mit hygienischen und roborierenden Maßnahmen behandelt. Nacb positivem Ausfall der W. R. spezifische Behandlung, wöchentlich $0 \cdot 1 \mathrm{ccm}$ Ol. cinereum und täglich $4 \mathrm{~g}$ 
Kal. jodat.; nach 2 Monaten Rückgang sämtlicher physikalischer (ahsolute Dämpfung links in der Axillarlinie von der 6.-10. Rippe, relative Dämpfung von der 10.-12. Rippe, mittelfeinblasiges Rasseln, Bronchialatmen) und klinischer Erscheinungen.

Besprechung der Differentialdiagnose gegenüber der Lungentuberkulose. Inkongruenz zwischen der Schwere der funktionellen Erscheinungen and dem physikalischen Befund, Lokalisation in den unteren Abschnitten, Fehlen des Fiebers, Nachweis sicherer syphilitischer Veränderungen an anderen Organen und der eventuelle Befund der Spirochaete pallida in Auswurf sprechen für Lnes.

Ledo, Antonio. Pyodermitis vegetans papillomatosa chronicamitatypischem Epithelwachstum (Azua).

52 Jahre alter Mann mit einer seit 14 Monaten bestehenden Affektion des $r$. Handräckens. 3 halbmondförmige Substanzdefekte mit geröteten infiltrierten Rändern, leicht blutenden Granulationen und seröseitriger Sekretion. Schnelle Heilung unter Sublimatverband und Kauterisation.

Die histologische Untersuchung ergab Verdickung und Abhebung des Stratum corneum durch ein fibrinöses Exsudat. Karyolytische Veränderungen und Bildung von Bläschen im Stratum germinativum, Infiltration der Epidermis mit Leukozyten, des Korium mit Leukozyten, Plasmazellen und Mastzellen. In der Tiefe des Korinm Inseln und Stränge von Epithel und Hornperlen, die in allen Fällen den Zusammenhang mit der Epidermis erkennen lassen.

Aja, Sainz de. Rezidivierende Aphthen der Mundhöhle.

Rericht über 3 Fälle, die wegen des positiven Ausfalles der W. R. als ätiologisch zusammengehörig angesehen werden, Spirillenerkrankungen. Alle 3 Fälle sahen klinisch nicht wie Lues aus, bei 2 Fällen war Lues anamnestisch auszuschlielßen, in dem 3. Fall bestand außer der Stomatitis aphthosa eine alte zur Zeit nicht manifeste Lues; in allen 3 Fällen. W. R. positiv. Vollkommen einwandsfrei, namentlich was die Wirksamkeit der Salvarsantherapie anlangt, erscheint nur 1 Fall; nach $0.6 g$ Neosalvarsan intravenös innerhalb von 48 Stunden vollkommene Heilung. Von den beiden anderen Fällen konnte der eine aus äußeren Gründen nicht mit Salvarsan behandelt werden und der andere war mit Lues kompliziert.

Schwierigkeit der Differentialdiagnose gegenüber der Lues, die noch vermehrt wird durch den bei beiden Krankheiten positiven Ausfall der W. R.

Azua, Juan de. Aplasia pilorum muniliformis.

Bericht über 3 Fälle dieser seltenen Erkrankung; es fanden sich die typischen Veränderungen der Kopfhaut - lichenartige Knötchen den Follikelmündungen entsprechend, atrophische Haut - und der Haare, in regelmäßigen Abständen wechselnde spindelförmige Verdünnungen und kugelige Verdickungen, starke Brüchigkeit.

Bei dem 1. Fall, einem jetzt 4 Jahre alten Mädchen, begannen die 
Veränderungen im 4. Monat. Sonst keine allgemeinen Erscheinungen, keine Heredität, Gebiß normal, Augenbrauen und Wimpern unverändert. Geringe Besserung mit Resorzin-Sublimatspiritus. Die beiden auderen Fälle betreffen 2 Schwestern von 5 und 9 Jahren. Die Mutter zeigt Symptome von $\mathrm{Bas}$ a $\mathrm{d}$ w und hat an den äußeren Dritteln beider Brauen pigmentlose Haare. Die Veränderungen bestehen angeblich von Geburt an, was als eine Ausnahme angeseben werden muB. Bei der älteren Schwester Lichen pilaris der Arme und pigmentlose Haare an den Brauen, sonst keine Allgemeinerscheinungen, Gebiß regelmäßig, Haare der Wimpern und Brauen im übrigen normal.

Die Therapie, Exzitantien, Kohlensäureschnee, Quarzlampe, Opotherapie mit Schilddrüsen- und Hodenextrakt, ohne Erfolg.

Ratera, Julián. Pruritus postekzematosus geheilt durch Radiotherapie.

1/2 Erythemdosis, 8 Tage Pause, Verschwinden des Juckens in 6 bis 7 Tagen.

Covisa, José S. Syphilitisches Neurorezidiv.

22jähriges Mädchen mit Lues I, intravenöse Salvarsaninjektion 0.45, sonst keine Behandlung. 33 Tage nach der Injektion heftige Kopfschmerzen, Ohrensausen, Schmerzen, Taubheit im r. Uhr, Fazialislähmung r., zerebrales Erbrechen, Teilnahmlosigkeit. Behandlung mit Hydrargyr. bicyanat. 0.01 täglich intravenös; nach der 4. Injektion Parästhesien im 1. Arm und Bein mit folgender kompletter motorischer Lähmung. Wegen beginnender Stomatitis Hg-Behandlung abgebrochen. Nach 4 intravenösen Injektionen von Neosalvarsan $0 \cdot 15$ Heilung.

Aja, Sainz de. Ein Fall von Hemichorea auf der Basis syphilitischer Gefäßerkrankung.

58 jähriger Mann, vor 35 Jahren Lues, die nur ungenügend mit Hg. und Jk. behandelt wurde; später nie mehr Frscheinungen der Lues und keine Behandlung. August 1912 obne jede Prodrome plötzliches Auftreten einer Hemichorea auf der ganzen l. Körperhälfte, Gesicht, Rumpf und Gliedmaben. Die Bewegungen verstärken sich bei willkürlicher Innervation und sistieren im Schlaf; keine Verminderung der groben Kraft. Keinerlei Allgemeinerscheinungen, wie Kopfschmerzen oder Erbrechen.

Geringe Besserung unter Schmierkur und Jod innerlich. Wesentliche Besserung, es blieben nur noch atethotische Bewegungen im Handgelenk und iden Fingergelenken zurück, durch intravenöse Injektionen von Hydrargyr. bicyanat 0.01-0.02 g.

Patient brach die Kur vorzeitig ab, da er sich für fgeheilt hielt. Nach 8/4 Jahren kam es unter Schwindel und Bewußtseinsverlust zu einem erneuten Auftreten der Hemichorea, der nach 4 Tagen eine vollkommene Hemiplegie der linken Seite folgte. Energische intravenöse Hg- und innerliche J.-Behandlung ohne wesentlichen Erfolg. Der Fall ist interessant wegen der langen Latenzperiode der Lues und der eigenartigen Lokalisation beim 1. Anfall. 
Rocamora, Jaime Peyri. Vorläufige Mitteilung zum Studium des Leukocytozoon syphilis.

Nachprũfung der Angaben von Mc. Donag h, British Journal of Dermatology, Nov. 1913, Jan. 1913; nach dessen Untersuchungen ist die Spirochaete pallida nicht die einzige morphologische Ausdrucksform des Syphiliserregers, sondern sie stellt nur ein Stadium, nämlich die männliche Sporozoite dar. Für diese Anschauung werden geltend gemacht die lange Inkubation, das intermittierende Wiederaufflackern, die ungenügenden Erfolge einer einmaligen Behandlung, das Fehlen von Teilungserscheisungen der Spirochaete und das seltene Vorkommen oder völlige Fehlen von Spirochaeten bei den tertiären Formen.

Die Entwicklung stellt sich folgendermaben dar: Infektion einer weißen Blutzelle; einer großen mononukleären, durch einen Sporozoit; in der Zelle entweder Teilung mit männlicher und weiblicher Differenzierung oder ohne Teilung Entwickiung nach dem männlichen Typus, Befruchtung, Bildung von Zygoten, Sporoblasten und Sporozoiten.

Das von R. untersuchte Material bestand aus 5 Sklerosen, 11 Drüsen aus verschiedenen Stadien sekundärer Lues und Drüsen von infizierten Kaninchen. Es wurde mit Azurmethylenblau, Giemsa und UnnaPappenheim gefärbt und ungefärbt im Ultramikroskop untersucht.

Außer den von Mc. Donagh beschriebenen Formen konnte eine birnenförmige Art der Sporozoite gefunden werden. Die Sporozysten und Sporozoite fanden sich am zahlreichsten in den Drüsen von mit Salvarsan behandelten Personen. Der Nachweis derselben gelingt leichter als derjenige der Spirochaeten.

Auch bei der experimentellen Kaninchensyphilis wurden dieselben Formen gefunden.

R. sieht in den von ihm bestätigten Befunden von Mc. Donagh einen wesentlichen Fortschritt in praktischer und theoretischer Beziehung.

Referiert nach den Actas Dermo-Sifiliográficas, año V. Nr. 3.

Hannes (Hamburg).

\section{Fachzeitsehriften.}

\section{Dermatologische Wochenschrift.}

Nr. $13-18$.

Nr. 13.

Hedén, K. Klinische Beobachtungen über die Einwirkung des Salvarsan und der Kombination ron Salvarsan und Quecksilber auf den Fötus. p. 362 . 